Herzschr Elektrophys 2013 $\cdot 24: 81-82$

DOI 10.1007/s00399-013-0258-6

Online publiziert: 3. April 2013

(c) Springer-Verlag Berlin Heidelberg 2013

Timo Weimar · Nicolas Doll

Sana Herzchirurgie Stuttgart GmbH, Stuttgart, Deutschland

\title{
Chirurgische Ablation von Vorhofflimmern
}

Patientengut mit VHF und einer Indikation für einen herzchirurgischen Eingriff bezüglich ihres Outcomes mit oder ohne begleitender Ablationstherapie evaluierte. Trotz dieser interessanten Fragestellung in einer klinisch relevanten Patientengruppe sind leider auch einige methodische Eckpunkte dieser Studie kritisch zu hinterfragen. Die Studie zeigte eine signifikant höhere Sinusrhythmusrate in der Ablationsgruppe überraschenderweise nur bei Patienten mit langanhaltendpersistierendem VHF, ausgerechnet bei der Subgruppe, die am schwierigsten zu therapieren ist. Insgesamt waren die Erfolgsraten mit $62 \%$ für paroxysmales, $72 \%$ für persistierendes und $52 \%$ für langanhaltend persistierendes VHF eher enttäuschend und widersprüchlich gegenüber der publizierten Literatur. Diese Rhythmusanalyse erscheint aber nur von relativ geringem Wert angesichts der Tatsache, dass mit dem epikardialen Einsatz von Kryoenergie ein Verfahren gewählt wurde, dass nachgewiesenermaßen eine deutliche Einschränkung der Linientransmuralität aufgrund des ausgeprägten Heatsink-Effekts mit sich bringt und das Follow-up lediglich aus einem 24-h-Langzeit-EKG nach einem Jahr bestand. Insgesamt konnte das klinische Ergebnis nach einem Jahr in dieser Studie durch die Ablation nicht verbessert werden.

In einer großen retrospektiven Studie mit 212 Patienten konnten Weimar et al. [3] zeigen, dass kein Unterschied in den Erfolgsraten zwischen der Cut\&Sew CoxMaze-III-Prozedur und der ablationsassistierten Cox-Maze-IV-Prozedur als Stand-alone-Verfahren bestand, während die prozedurassoziierte Morbidität signifikant gesenkt werden konnte. Eine Kaplan-Meier-Analyse ergab eine Freiheit von symptomatischem $\mathrm{VHF}$ von $85 \%$ nach 10 Jahren. In der kompletten Serie wurden nur 2 Schlaganfälle (0,9\%) während des gesamten Follow-ups dokumentiert. Pet et al. [4] zeigten weiter, dass ein hoher $\mathrm{CHADS}_{2}$-Score keinen Einfluss auf das Auftreten neurologischer Ereignisse nach einer Cox-Maze-Prozedur hat und das jährliche Risiko für Schlaganfälle nach einer solchen mit $0,2 \%$ erstaunlich niedrig ist (433 Patienten, $32 \%$ mit einem $\mathrm{CHAD}_{2}$-Score $\geq 2$ ).

Mark La Meir [5] veröffentlichte Ergebnisse eines Hybridverfahrens, in dem eine epikardiale endoskopische Ablation mit einem endokardialen Mapping und ggf. Ablation durch den Elektrophysiologen in gleicher Sitzung kombiniert wird. Mit diesem Verfahren konnten Erfolgsraten von $93 \%$ für paroxysmales und $90 \%$ für persistierendes Vorhofflimmern erreicht werden. Interessanterweise konnten dabei intraoperativ bei $23 \%$ der Patienten Lücken in den Ablationslinien, v. a. in der superioren Konnektionslinie zwischen den oberen rechten und linken Pulmonalvenen, detektiert werden. Ein Vergleich zwischen alleiniger endoskopischer Ablation und einem Hybridverfahren bei 63 Patienten ergab signifikant bessere Erfolgsraten für den Hyprid-Ansatz nach einem Jahr (100 vs. 87,5\%, $p=0,04)$.

Die Autoren konnten bei 89 Patienten mit dem endoskopischen Zugang eine Erfolgsrate von 88,90 und $90 \%$ nach 6,12 und 24 Monaten erreichen, ohne signifikanten Unterschied zwischen paroxysmalem und persistierend/langanhaltend persistierendem VHF [6]. Die Lebensqualität war 12 Monate nach dem Eingriff für alle Domänen des Medical Outcomes Study 36-Item „Short-Form Health Survey“ signifikant verbessert gegenüber den präoperativen Ausgangswerten $(p<0,001)$. 


\section{Studien-Highlights 2012}

\section{Korrespondenzadresse}

\section{Dr. T. Weimar}

Sana Herzchirurgie Stuttgart GmbH

Herdweg 2, 70174 Stuttgart

timo.weimar@yahoo.de

Interessenkonflikt. Der korrespondierende Autor gibt für sich und seine Koautoren an, dass kein Interessenkonflikt besteht.

\section{Literatur}

1. Budera $P$, Straka Z, Osmancik $P$, Vanek T, Jelinek $S$, Hlavicka J, Fojt R, Cervinka P, Hulman M, Smid M, Maly M, Widimsky P (2012) Comparison of cardiac surgery with left atrial surgical ablation vs. cardiac surgery without atrial ablation in patients with coronary and/or valvular heart disease plus atrial fibrillation: final results of the PRAGUE-12 randomized multicentre study. Eur Heart J 33(21):26442652

2. Boersma LV, Castella M, van Boven W, Berruezo A, Yilmaz A, Nadal M, Sandoval E, Calvo N, Brugada J, Kelder J, Wijffels M, Mont L (2012) Atrial fibrillation catheter ablation versus surgical ablation treatment (FAST): a 2-center randomized clinical trial. Circulation 125:23-30

3. Weimar T, Schena S, Bailey MS, Maniar HS, Schuessler RB, Cox JL, Damiano RJ Jr (2012) The coxmaze procedure for lone atrial fibrillation: a singlecenter experience over 2 decades. Circ Arrhythm Electrophysiol 5:8-14

4. Pet M, Robertson JO, Bailey M, Guthrie TJ, Moon MR, Lawton JS, Rinne A, Damiano RJ Jr, Maniar HS (2012). The impact of CHADS (2) score on late stroke after the Cox maze procedure. J Thorac Cardiovasc Surg [Epub ahead of print]

5. La Meir M, Gelsomino S, Luca F, Pison L, Parise $\mathrm{O}$, Colella A, Gensini GF, Crijns H, Wellens F, Maessen JG (2012) Minimally invasive surgical treatment of lone atrial fibrillation: early results of hybrid versus standard minimally invasive approach employing radiofrequency sources. Int J Cardiol [Epub ahead of print]

6. Weimar T, Vosseler M, Czesla M, Boscheinen M, Hemmer WB, Doll KN (2012) Approaching a paradigm shift: endoscopic ablation of lone atrial fibrillation on the beating heart. Ann Thorac Surg 94(6):1886-1892 